\title{
Análise da prática docente com rede social educativa na disciplina de Programação Orientada a Objeto
}

\author{
Elias Vidal Bezerra Junior ${ }^{1}$, Alex Sandro Gomes ${ }^{2}$, Flávia Veloso Costa Souza ${ }^{3}$, \\ Socorro Vânia Lourenço Alves ${ }^{2}$ \\ ${ }^{1}$ Instituto Federal de Educação, Ciência e Tecnologia do Tocantins (IFTO) \\ Porto Nacional - TO - Brasil \\ ${ }^{2}$ Centro de Informática/Universidade Federal de Pernambuco (CIN/UFPE) \\ Recife - PE - Brasil \\ ${ }^{3}$ Departamento de Ciências Exatas/Universidade Federal da Paraíba (DCE/UFPB) \\ Rio Tinto - PB - Brasil \\ $\{$ evbj, asg, fvcs, svla\}@cin.ufpe.br
}

\begin{abstract}
This paper analyses the practice of teaching object-oriented programming technology mediated by a social networking educational Redu. The aim of the study was to describe the teaching practices and obtain a qualitative assessment of their effectiveness. It was used a qualitative method with interviews, observations and comments online. The results show evidence of effective practice in relation to actions and teaching activities.
\end{abstract}

Resumo. Este artigo analisa a prática de ensino de programação orientada a objeto mediado por tecnologia de rede social educativa Redu. O objetivo do estudo foi descrever as práticas docentes e obter uma avaliação qualitativa de sua efetividade. Foi adotado um método qualitativo com entrevistas, observações e observações online. Os resultados mostram indicios de uma prática efetiva e bem estruturada em termos de ações e das atividades docentes.

\section{Introdução}

No contexto acadêmico o ensino de programação e modelagem através de métodos orientados a objetos tornou-se uma prática comum na introdução da Ciência da Computação durante os últimos dez anos [Anquan et al., 2012]. A Programação Orientada a Objeto (POO) segundo Li e Xu (2010) é uma maneira de pensar sobre o processo de decompor o problema e desenvolver soluções de programação.

Apesar da ampla adesão à POO, algumas pesquisas indicam que iniciantes em programação têm dificuldades na compreensão e aplicação de conceitos e abstrações presentes nesse paradigma [Xinogalos et al., 2006; Holland et al., 1997]. Isso tem contribuído para a geração de um alto índice de evasão e reprovação nas disciplinas que requerem conhecimentos de POO [Hinterholz Jr., 2009]. 
Com o intuito de apoiar o ensino de POO várias abordagens e ferramentas têm sido propostas, como o ensino baseado em jogos [Rais et al., 2011], a criação de metodologias de ensino de POO como o iPOO [Lopes, 2007] e o modo de ensino de aprendizagem em ciclo [Hong e Wang, 2009]. A análise das soluções mais recentes mostra que existe uma tendência em colocar o aluno no centro do processo ensinoaprendizagem, transformando-o em um sujeito ativo. Nessa direção, Li et al. [2009] aponta que as metodologias de ensino de POO devem colocar o aluno como sujeito ativo do seu processo de aprendizagem.

Seguindo a perspectiva de Li et al. [2009] as redes sociais baseadas na Web apresentam-se como meios para que os fenômenos de colaboração e interação ocorram de forma mais efetiva. Em particular, as redes sociais virtuais voltadas para a educação são espaços nos quais o professor pode criar uma rede de relacionamentos e um novo canal de comuniação com seus alunos, onde todos podem colaborar, trocar ideias e recursos sobre determinado conteúdo.

Neste estudo, buscamos indícios que nos permitam concluir se o uso de uma rede social educativa virtual pode proporcionar uma prática de ensino de $\mathrm{POO}$ efetiva. $\mathrm{O}$ estudo foca especificamente a prática do docente dentro desses ambientes digitais, visto que a ação docente é um fator primordial no processo de ensino-aprendizagem [Gadotti, 2000]. Partimos da hipótese que as redes sociais educativas podem apoiar o docente no ensino de POO, já que estas possibilitam a exposição dos conteúdos, a colaboração e a interação social entre os usuários, o compartilhamento de materiais, dentre outros recursos. A análise foi realizada dentro da Rede Social Educacional REDU.

O presente artigo está estruturado conforme descrito a seguir. A seção 2 apresenta o uso de redes sociais na educação. A seção 3 apresenta o REDU. Na seção 4 será apresentada o método utilizado no estudo. A seção 5 apresenta os resultados e, por fim, a seção 6 apresenta as conclusões.

\section{O uso de redes sociais na educação e a prática docente}

As redes sociais baseadas na Web surgiram em meados da década de 90, porém nos últimos anos, elas têm apresentado um crescimento rápido tanto no número de usuários quanto em seus propósitos - como o compartilhamento de vídeos, comércio, ensino, entre outros. Uma vantagem das redes sociais virtuais é que elas permitem formas de interações ricas, não lineares e plurais, visando promover a colaboração e disseminação de conteúdo em círculos sociais motivados [GOMES et al., 2012].

Dentro do contexto educacional algumas pesquisas [Cho et al., 2007; Haythornthwaite, 2002; Melo, 2010; Silva e Cogo, 2010] apontam as estruturas das redes sociais como ambientes colaborativos de aprendizagem que podem favorecer o desenvolvimento dos aprendizes. Segundo Silva e Cogo (2007), esses novos ambientes digitais vem ampliando a colaboração, a interatividade e a flexibilidade de tempo e espaço de sala de aula, por isso é possível fazer uso das redes sociais para contribuir no processo de ensino-aprendizagem.

Queiroz (2011) ressalta que a partir de um estudo exploratório em anais de eventos nacionais e internacionais, sobre o uso das redes sociais no contexto educacional, ocorridos no Brasil a partir de 2005, foram encontrados vários estudos 
sobre experiências realizadas com a utilização das redes sociais virtuais (como Facebook, Orkut, Twitter, entre outros) e pesquisas com diferentes propósitos sobre essa temática.

No entanto, apesar da existência de várias iniciativas dessas redes na educação, observa-se que em muitos casos os docentes ainda apresentam dificuldades em fazer uso dessas plataformas como ferramenta de suporte ao ensino (Kenski, 2003; Queiroz, 2010), uma vez que o uso destas implica em uma mudança na prática docente.

Claudeivan (2011) destaca o fato de a metodologia usada pelo docente afetar diretamente a aprendizagem do estudante, podendo a mesma influenciar positivamente, ou não, a construção do seu conhecimento. A prática docente está relacionada à realização de três atividades, sendo elas: a atividade prévia de planejamento, atividade de ensino propriamento dito, que é conhecido como a fase ativa; e a atividade de avaliação do ensino [Tardiff e Lessard, 2008].

Neste estudo, conforme apresentado na seção anterior, analisamos a prática docente dentro da Rede Social Educacional REDU ${ }^{1}$, apresentado na próxima seção. $\mathrm{O}$ objetivo deste estudo foi avaliar, através de uma análise qualitativa, se o uso de uma rede social educativa virtual pode proporcionar uma prática de ensino de POO efetiva.

\section{Rede Social Educacional - REDU}

O REDU é uma rede social educativa desenvolvida para servir de suporte a fenômenos de colaboração e aprendizagem [GOMES et al, 2011]. O ambiente apresenta um novo conceito de plataforma de ensino, utilizando as potencialidades das redes sociais, que busca aproximações com o cotidiano dos usuários, oferecendo uma interface com acesso simplificado e intuitivo. Conforme citado na seção 1, foi escolhida neste trabalho por refletir as estruturas da prática docente, desde o planejamento, passando por processos de mediação da aprendizagem, até as atividades de monitoramento e avaliação [GOMES et al., 2012].

Com finalidade educativa, o REDU, dispõe de ferramentas para mediar a comunicação e a colaboração em rede, como também, oferecer recursos para planejamento de atividades, compartilhamento de materiais didáticos, coordenação, (auto)avaliação e (auto)regulação da aprendizagem (GOMES et al., 2012). Por meio de sua plataforma, alunos, professores e instituições têm a possibilidade de tornar a aprendizagem um processo contínuo que vai além dos tempos e espaços das instituições de ensino. Segundo Melo (2010), o REDU possui ferramentas capazes de proporcionar ao processo de ensino e aprendizagem uma dinâmica maior na relação professor/professor, professor/aluno e aluno/aluno. Dentre as ferramentas destacamos o mural que é o núcleo principal de comunicação dentro da plataforma. Através dele, docentes e estudantes podem postar comentários, respostas e pedidos de ajuda. É importante também ressaltar que o REDU viabiliza o uso de Recursos Educacionais Abertos, através de uma API (Application Programming Interface, ou Interface de Programação de Aplicativos). A documentação da API do REDU encontra-se disponível em HTTP://developers.redu.com.br, e tem a finalidade de possibilitar a integração de

\footnotetext{
${ }^{1}$ www.redu.com.br
} 
forma simples e transparente de software educacionais no REDU para uso de seus usuários.

\section{Método}

Este trabalho adota uma abordagem qualitativa, a partir da realização de estudo de caso com professores da disciplina de Programção Orientada a Objeto do $4^{\circ}$ período do curso de Licenciatura em Ciência da Computação do Instituto Federal de Educação, Ciência e Tecnologia do Tocantins - campus Porto Nacional, por um semestre letivo. Durante o estudo foi realizada observação participante e realizadas entrevistas não-estruturada com os professores envolvidos. A disciplina de POO foi ofertada na modalidade mista de ensino, blended learning, sendo intercalada por momentos presenciais e momentos de colaboração a distância. A disciplina teve uma carga horária de 60 h.a. As aulas presenciais tiveram uma duração de 2 horas cada aula, e ocorriam duas vezes na semana. Nos intervalos entre as aulas presenciais foram realizadas discussões a distância por meio do REDU. Estas objetivavam elucidar dúvidas e discutir conteúdos relacionados à disciplina, nesses momentos também eram realizadas atividades práticas propostas pelos docentes.

\subsection{Coleta dos dados}

A coleta dos dados ocorreu através da filmagem e gravação dos momentos presenciais e da geração de todos os logs de acesso nos momentos a distância. Todos os participantes, docentes e discentes, foram comunicados e autorizaram a coleta dos dados.

A observação dos docentes foi realizada em três momentos, que foram: a observação do planejamento da disciplina pelos docentes, considerando o uso do REDU e a inserção de todos os módulos e atividades pelo docente dentro do ambiente; a observação das aulas presenciais (ocorridas em sala de aula e no laboratório) e o acompanhamento dos momentos a distância através do mural da disciplina, ou seja, a interação do professor com os estudantes, mediado pelo REDU; e por fim a avaliação dos alunos pelos docentes. A observação do uso do REDU foi realizado por meio de captura de telas, gravações e anotações.

\subsection{Procedimentos}

Em um primeiro momento foram definidos os locais e os sujeitos da pesquisa. A partir de visitas realizadas ao Instituto Federal de Educação e conversas com os docentes os mesmos foram convidados para participar da pesquisa. Escolhemos dois docentes da mesma disciplina.

Após a escolha dos docentes foi realizada a fase de preparação das atividades. Durante esta etapa foi realizado o treinamento dos docentes para a utilização do REDU. Nesta etapa, também foi realizado o acompanhamento das atividades de planejamento dos docentes para a disciplina. Durante o planejamento os docentes fizeram uma préseleção dos recursos que seriam utilizados na plataforma.

A etapa seguinte consistiu no acompanhamento da disciplina. Inicialmente, os alunos receberam um treinamento para uso da plataforma. Ao término desta etapa foi realizada uma análise de todos os dados coletados. 
Os docentes fizeram uso do Recurso Educacional Compilr ${ }^{2}$ no REDU; um ambiente de desenvolvimento online e também compilador para várias linguagens de programação. A Figura 1 ilustra o editor de programação Compilr disponibilizado através da API do REDU para realização das atividades na plataforma. O uso deste recurso permitiu ao aluno visualizar, manusear o conteúdo da disciplina e programar sem a necessidade de recorrer ou instalar uma IDE (Interface de Desenvolvimento) específica.

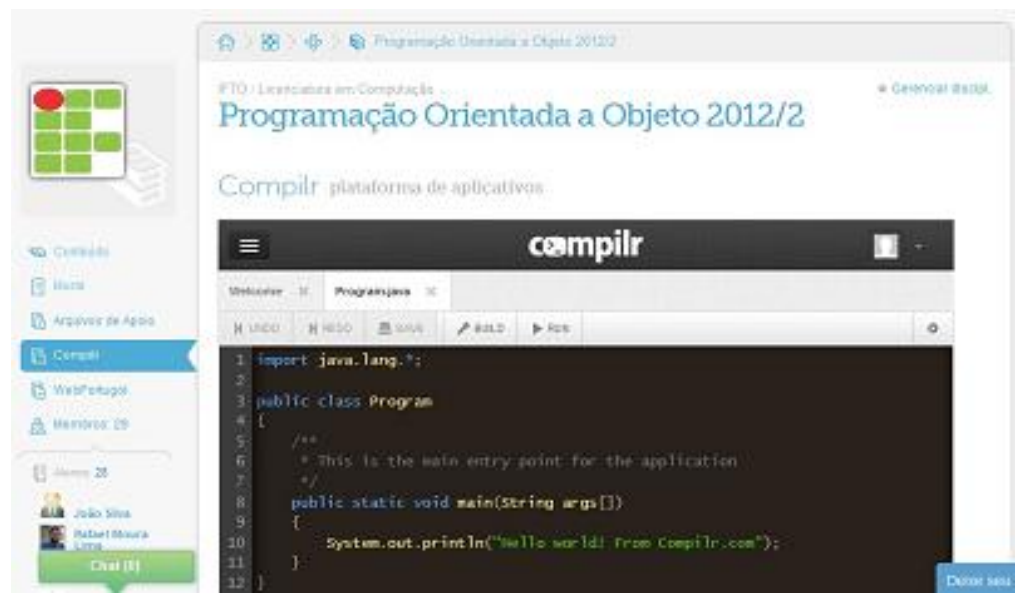

Figura 1 - Editor de programação Java disponibilizado através da API no REDU.

\subsection{Análise dos dados}

Para realizar a análise dos dados foram utilizadas as transcrições dos dados visuais e não visuais coletados por meio das técnicas de captura de telas do REDU, das entrevistas e observações descritas acima. Na análise da prática docen no ensino de POO no Redu foi feita a identificação das categorias. Cada categoria representava atividades realizada pelo docente. Após essa etapa, foi realizada a codificação dos dados. A análise e interpretação dos dados foram realizadas buscando descrever e interpretar as características do uso e os problemas ao usar a plataforma durante a prática docente no ensino de POO.

\section{Resultados}

Nesta seção serão descritas e discutidas as categorias identificadas na prática docente. Foram identificados vários episódios que envolvem as atividades do docente: desde os recursos utilizados, assim como, o planejamento, o monitoramento e a avaliação no processo de ensino-aprendizagem. As categorias criadas descrevem a prática docente e constituem-se em provas que ajudam a reconhecer a efetividade do uso de uma rede social educativa no exercício da docência. As categorias apontadas no processo de análise da prática docente em POO, que destacaremos no presente artigo são: planejamento, ensino, avaliação e distância transacional.

\footnotetext{
${ }^{2}$ https://compilr.com
} 


\subsection{Planejamento}

Durante a etapa de planejamento da disciplina os professores realizaram atividades de estruturação da disciplina, organização das tarefas de aprendizagem, elaboração e pesquisa de materiais didáticos para serem utilizados em suas aulas. Essas atividades contemplavam tanto os momentos presenciais quanto os momentos a distância.

Segundo os professores, o uso da REDU apoiou a etapa de planejamento de forma efetiva, visto que eles tiveram a possibilidade de colaborar entre si, trocando ideias e experências que contribuíram para o planejamento da disciplina. A viabilização de diálogo entre os docentes favoreceu a integração entre as diferentes propostas de ensino, permitindo um professor apropriar-se de boas práticas de outros.

O recurso do tipo Aplicativos, disponível no REDU, também foi muito útil. Através dessa funcionalidade o professor pôde buscar ferramentas que auxiliaram na execução de atividades mais práticas, o que permitiu aproximar o aluno dos conceitos abstratos do paradigma orientado a objeto, como por exemplo, o professor usou um objeto de aprendizagem (OA) que apresentou de forma animada os conceitos de classe e objeto e a sintaxe como estes conceitos devem ser implementados na linguagem JAVA.

A funcionalidade do REDU de dividir o conteúdo em módulos e estes em aulas com materiais associados, permite ao docente ter uma visão sequencial de todo o plano de ensino. Cria-se assim uma rota de aprendizagem que consiste na estruturação dos cenários de ensino a serem percorridos pelo aluno no decorrer das aulas, estabelecendo uma comunicação tridimensional entre aluno-professor-conhecimento. Os professores puderam também ajustar as atividades dos módulos e adicionar novos recursos didáticos, à medida que acompanhavam o desempenho dos alunos.

A seguir será transcrito alguns protocolos com falas dos professores em relação à categoria de planejamento da disciplina de POO:

P2: "Gostei de organizar no inicio a disciplina no redu porque a possibilidade de disponibilização do conteúdo de forma sequencial, seguindo a ementa da disciplina, ajudou a ter uma visão geral da disciplina"...

P2: ... “ Foi muito bom elaborar o planejamento e ao mesmo tempo ter a oportunidade de conversar com o professor P1 pra gente trocar ideias sobre como oferecer a disciplina de modo que ajudasse mais os alunos... essa disciplina tem um indice de reprovação alto, trocar ideias com o colega aqui na rede foi mais fácil..."...

P1: ... " Foi muito bom ter a possibilidade de apresentar um conceitos de forma diferente, o uso dos objetos de aprendizagem nos permite diversificar a estratégia de ensino, dá ao aluno uma outra forma de enxergar e entender um conceito tão abstrato ... "...

\subsection{Ensino}

Adotando o mesmo padrão de outras redes sociais, o REDU disponibiliza aos professores e alunos murais como principais ferramentas de comunicação, mediação e participação. Através deles, alunos e professores interagiram de forma dinâmica e não linear, usando texto, imagem, vídeo e áudio. A partir das publicações nos murais os professores tiveram a oportunidade de perceber e acompanhar o processo de construção do conhecimento ocorrido na disciplina de POO e intervir nos momentos necessários. 
Os professores indicaram que o REDU ampliou o tempo da sala de aula. Para eles, através da interação no REDU, conteúdos mais complexos puderam ser explorados durante um maior tempo, favorecendo o aprendizado dos alunos com maiores dificuldades. Além disso, alunos mais habilidosos com programação também puderam compartilhar suas experiências com outros colegas, ajudando o professor na condução dos conteúdos. Essa facilidade, segundo os professores, propiciou a democratização do conhecimento.

Os professores acharam muito positivo a possibilidade da execução de códigos de programação dentro da própria rede social. Para eles, isso favoreceu bastante a configuração de diferentes cenários que davam suporte ao entendimento dos conceitos explorados. Entretanto, os docentes sentiram falta da possibilidade de visualizar, nos momentos a distância, as atividades que os alunos realizavam no compilador. Eles destacaram a necessidade da opção de colaboração para a realização dessas atividades.

A seguir será transcrito alguns protocolos dos professores em relação à categoria de ensino da disciplina de POO:

P2: "No Redu percebi que os alunos participavam mais, perguntavam mais também, não era só a sala de aula o momento de aprendizado. Um aluno chegou a comentar na aula que estava gostando porque ele na sala nunca conseguia tirar todas as dúvidas, por timidez... Até porque o tempo da aula passa rapidinho (...)”.

P2: "Muito bom a opção de abrir um compilador Java dentro da Rede através da plataforma de aplicativos, foi bom também para os alunos (....) agora seria melhor se a gente pudesse ver o que os alunos estavam fazendo e ajudar, isso só acontecia na sala"...

P1: "Senti falta de acompanhar o que os alunos programam! Seria bom se agente pudesse compartilhar o mesmo código..."

\subsection{Avaliação}

A avaliação da aprendizagem do aluno, por meio do REDU, ocorreu a partir do acompanhamento das participações nos murais da disciplina, através dos quais foi possível identificar o desempenho e as dificuldades de cada aluno. No REDU o professor também pôde elaborar testes objetivos para mensurar o aprendizado dos alunos. Assim, o professor teve a possibilidade de realizar um acompanhamento contínuo do processo de aprendizagem dos conteúdos dos seus alunos.

O REDU dispõe de um mecanismo de avaliação que ofereceu ao docente uma visão geral do desempenho da turma em relação aos conteúdos da disciplina (Figura 2). A ferramenta apresenta ao professor a quantidade de alunos que realizou a atividade, a média geral da turma, o tempo médio dispendido pelos alunos na execução da atividade e os melhores desempenhos obtidos. A posse dessas informações permitiu que os professores refletissem sobre seus objetivos e estratégias, levando a mudanças no planejamento ou na elaboração de atividades para apoiar o aprendizado do aluno. 

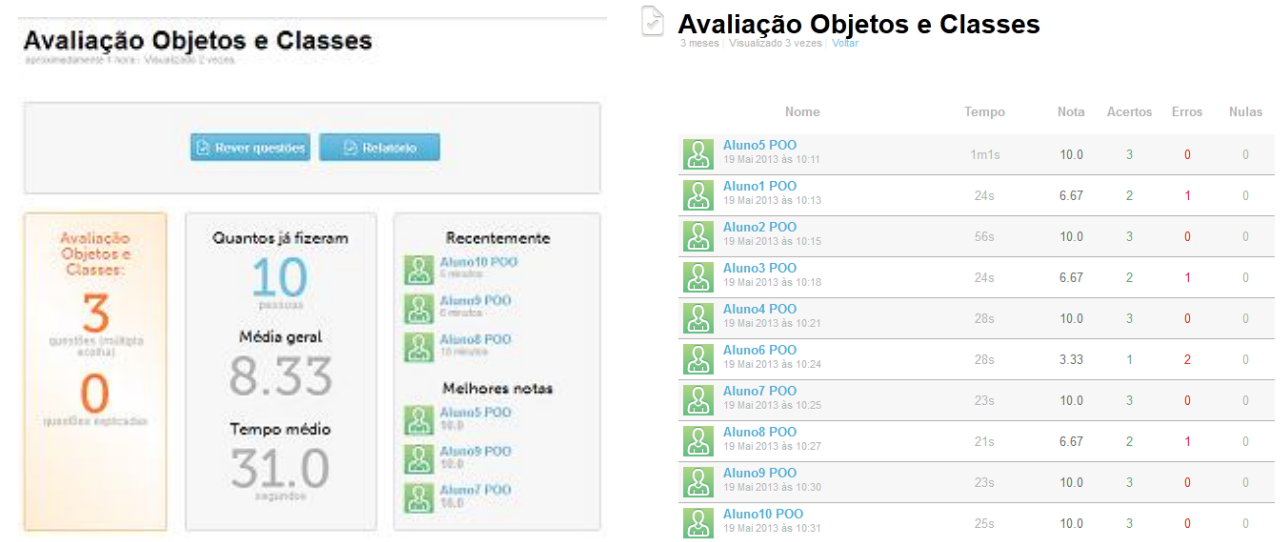

Figura 2 - Interação na Avaliação.

A seguir apresentamos um protocolo no qual o professor relata como foi o uso da ferramenta de avaliação:

P1: "Coloquei no Redu uma atividade de polimorfismo. Depois que olhei os resultados percebi que muitos alunos ainda estavam com dificuldade, inclusive a média geral foi abaixo de 6,0. Então achei melhor mudar um pouco o planejado ... então aumentei o número de aulas sobre o assunto e pesquisei mais material para colocar no Redu ..."

\subsection{Distância Transacional}

O conceito de distância transacional descreve a relação entre professor e alunos quando separados espacialmente, e foi construído baseado na estrutura do curso, na interação/diálogo entre os envolvidos e no nível de autonomia dos alunos no curso [MOORE, 2007; TORI, 2008]. Observamos que durante o estudo realizado na rede social educacional Redu, a forma como o curso foi estruturado e a interação entre os envolvidos fez com que a distância transacional fosse minimizada e a aprendizagem dos alunos maximizada.

Um exemplo pode ser visto no episódio descrito a seguir, onde um dos professores participantes destacou a colaboração que ocorreu entre os alunos e ele. Além da maior colaboração, também foi possível observar que durante o uso da rede os alunos sentiamse mais próximos do professor. A rede propiciou tanto um tempo maior para conversar e tirar dúvidas sobre os conteúdos, como também uma maior proximidade do professor com alunos, levando os alunos a enxergarem o professor como mais um integrante de seu grupo.

P1: "Pela interação em uma rede social eles puderam colaborar entre si. E ter a disposição 24 horas um material complementar as aulas presenciais. Também percebi que os alunos perguntavam mais, conversavam mais comigo e com os colegas. Foi uma experiência muito legal para eles e para mim. ....

Em vias de conclusão e a partir dos dados coletados, há indícios para afirmar que o ensino através de rede social educacional permitiu reduzir a distância transacional e motivar um maior nível de envolvimento e entre o professor e os alunos. 


\section{Conclusões}

O presente artigo teve como objetivo apresentar indícios que nos permitam concluir se o uso de uma rede social educativa virtual pode proporcionar uma prática de ensino de POO efetiva. Concluímos que o uso de uma rede social educativa apresenta-se como uma boa opção no processo de planejamento, ensino, avalição e aproximação da distância transacional dos conteúdos programáticos de programação orientada a objeto. Isso pode estar associado ao fato de a mesma ser transparente à realização de práticas de comunicação, proporcionar ajuda mútua, simplificação na forma de comunicação verbal. Esse conjunto de características favorecem a aprendizagem colaborativa e mediação didática docente no contexto de uma rede virtual de ensino. Ela parece ajudar a promover a reflexão sobre a prática docente e mudanças na relação professor e aluno, construindo assim novas relações sociais.

A criação de um ambiente virtual de ensino de programação orientada a objeto, através de uma rede social educacional, no qual professores passam a ser mediadores e alunos podem ser responsáveis pela construção do seu conhecimento: melhora o processo de ensino e aprendizagem, incentiva a pesquisa, propaga o conhecimento, ultrapassa barreiras da tradicional sala de aula, situa o recurso tecnológico como material de ensino, valorizando o papel fundamental do docente.

\section{Referências}

Anquan, J.; Yuqing, L.; Bailiang, C.; Jihua, Y.; Jie, Z. (2010), "The Education Reform and Innovation of Object oriented Programming Course in Normal University", The 5th International Conference on Computer Science \& Education. IEEE. Computer Science \& Education, Hefei, China. August 24-27, 2010

Cho, H., Gay, G., Davidson, B., e Ingraffea, A. R. (2007). Social networks, communication styles, and learning performance in a cscl community. Computers \& Education, 49(2), p. 309-329.

Claudeivan, L. C. (2011). Análise das práticas docentes de planejamento e mediação em redes sociais no ensino médio. Dissertação (mestrado profissional). Universidade Federal de Pernambuco, CIn, Pós graduação em Ciência da Computação, Recife PE.

Gadotti, M.(2000). Perspectivas atuais da educação. Porto Alegre: Ed. Artes Médicas.

Gomes A. S.; Souza, F. V. C.; Abreu, J. A. B.; Lima L. C. C.; Melo, C. A.; Paiva, G. L.; Duarte, A. P. (2011), "Colaboração, Comunicação e Aprendizagem em Rede Social Educativa", In Xavier A. C. (Ed.) Hipertexto e Cibercultura: links com a literatura, a publicidade, o plágio e as redes sociais educacionais, São Paulo: Respel.

Gomes, A. S., Rolim, A. L., Silva, W. M. (Eds.). (2012), "Educar com o Redu”, Recife: Redu Educational Technology, 103 p. ISBN 978-85-415-0037-1 Recife: Redu, Educational Technology.

Haythornthwaite, C. (2002). Building social networks via computer networks: Creating and sustaining distributed learning communities. In K. A. Renninger \& W. Shumar (Eds.), Building virtual communities: learning and change in cyberspace (p. 159-190). Cambridge: Cambridge University Press. 
Holland, S. Griffihs, R., Woodman, M., "Avoiding object misconceptions", ACM SIGCSE Bulletin, Vol. 29, No. 1, 1997, pp. 131-134.

Kenski, V. M. (2003). Tecnologia e as Alterações no Espaço e Tempo de Ensinar e Aprender. São Paulo: Papirus.

LI, Longshu, XU, Yi. 2010. The Teaching Research on a Case of Object-Oriented Programming. The 5th International Conference on Computer Science \& Education. IEEE.

Liu, L. Liu, J. Zhuang, H. Zhaoqing, W.. (2009), "LCM Exploration and Practice", In OOP Teaching. Scalable Computing and Communications; Eighth International Conference on Embedded Computing, 2009. SCALCOM-EMBEDDEDCOM'09. International Conference on. IEEE. 25-27 Sept. 2009.

Lopes, Mauricio Capobianco. iPOO: uma metodologia para o ensino introdutório de orientação a objetos. Seminário de Informática - RS (SEMINFO RS'2007). I WEI Tchê - Workshop sobre Educação em Informática.

Melo, C. A. Scaffolding of Self-Regulated Learning in Social Networks, Dissertação (Mestrado em Ciência da Computação) — Universidade Federal de Pernambuco, 120 p., 2010.

Moore, M. (2007). Educação a distância: uma visão integrada. Michael G. Moore, Greg Kesrley. Tradução Roberto Galman. São Paulo: Thomson Learning.

Queiroz, D. N. (2010). Desenvolvimento de um componente de visualização de informação para a plataforma de ensino a distância Amadeus. Trabalho de Conclusão de Curso (Graduação em Ciência da Computação), 51 p., Universidade Federal de Pernambuco, Recife - PE.

Queiroz, T. L. A (2011). Redes Sociais: a concepção dos professores sobre as possibilidades de uso na Educação. X Congresso Nacional de Educação - I Seminário Internacional de Representações sociais, subjetividade e educação - SIRSSE. Curitiba/PR.

Rais, Aimi Elliyana, Sulaiman, Shahida, Syed-Mohamad, Sharifah Mashita. 2011. Game-based Approach and its Feasibility to Support the Learning of Object-Oriented Concepts and Programming. Malaysian Conference in Software Engineering (MySEC). IEEE.

Silva, A. P. S. S.; Cogo, A. L. P. (2007). Aprendizagem de punção venosa com objeto educacional digital no curso de graduação em enfermagem. Revista Gaúcha de Enfermagem. Porto Alegre/RS, v. 28, n. 2, p.185-192.

Tardiff, M. e Lessard C. O. (2008) Trabalho Docente-Elementos para uma Teoria da Docência como Profissão de Interações Humanas. Petrópolis/RJ: Editora Vozes.

Tori, R. (2008). Cursos híbridos ou blended learning. In: Educação a distância: O Estado da Arte, Ed. São Paulo: PEARSON.

Xinogalos, S., Sartatzemi, M., Dagdilelis, V., Evangelidis, G. (2006). Teaching OOP with BlueJ: A case study. Proceedings of the Sixth International Conference on Advanced Learning Technologies (ICALT'06), IEEE. 